As had been previously shown, the percentage of Staphylococci resistant to prevalent antibiotics remained high, namely, 70 per cent, though they were uniformly sensitive to erythromycin. Resistance of Escherichia coli, from cases of urinary infection, to sulphonamides, streptomycin and tetracycline was found to be on the increase.

Work on the survival of smallpox virus in a nonpathogenic form in the brain of infant mice is in progress with the view of finding the $L D_{50}$ in different passage-levels of the variola virus. Studies are still being carried out to determine the normal antibody titre in random population samples, and especially among young children, against variola virus.

Leucoderma skins receiving ultra-violet radiation, compared with a non-irradiated control, on histological examination showed an increase of melanocytes with proliferation of dendrites and abundance of melanin. To substantiate previous observations that counter-irritant drugs, commonly applied locally, are apt to produce hard erythematous and reactionary patches, ultimately resisting any form of treatment, a number of patients with leucoderma were treated with psoralen ointment in a soothing base. The results have been encouraging, and further work is in progress.
Histamine reaction in cases of non-lepromatous leprosy showed significant quantitative difference when compared with that on the normal skin. Studies on serum protein in the two polar types of leprosy revealed an increase of $\gamma$-globulin fraction in sera of lepromatous cases. Formation of leishmanial granuloma was demonstrated in the spleen of drugresistant kala-azar. Biochemical observations of interest were made on the liver of animals infected with Trypanosoma evansi.

A new species of Dioscorea yams, obtained from the Darjeeling area, was found to contain as much as 4 per cent diosgenin. The glycoside content of Digitalis was found to reach its maximum when the plant was about $1 \frac{1}{2}$ years old. Three compounds of indigenous drugs were prepared for clinical trial, namely, arborine hydrochloride, aegelin and psoralen. A volatile oil derived from the seeds of Nigella sativa was shown to have a mosquitocidal property; its chemical composition was determined. Four new compounds were synthesized for possible use as insecticides and insect repellants.

A number of new drugs was also subjected to clinical trial to evaluate their therapeutic efficacy, particularly against amobiasis, leishmaniasis, helminthiasis, and skin diseases, including superficial mycosis and leprosy.

\title{
CANCER SERVICES IN EUROPE
}

A PAPER prepared by Dr. A. Chaklin, chief of the Cancer Division, World Health Organization, Headquarters, Geneva, and Dr. J. Mosbech, Office of Medical Statistics, Danish National Health Service, served as a background document for the technical discussions held during the eleventh session of the World Health Organization Regional Committee for Europe at Luxembourg in September 1961. The main points have been brought together in an article in the WHO Chronicle (16, No. 3; March 1962). While the ætiology of most forms of cancer is still unknown, many of them can be effectively treated. For this, well-organized services are essential. There are considerable differences in the organization of cancer control in the various countries of Europe. In the Scandinavian countries cancer falls within the province of the family doctor and the general hospital. In the United Kingdom, also, there is no separate speciality for malignant disease, all treatment being carried out on a regional basis within the ordinary facilities of the National Health Service. There are, however, certain hospitals and clinics reserved for cancer treatment, and a number of homes for the care of incurable cases.

The situation in the U.S.S.R. and the countries of eastern Europe is quite different. A network of cancer services with a high degree of independence, staffed by doctors especially trained in the detection, treatment and prevention of the disease, was established by Government decree in the U.S.S.R. after the Second World War.

This network consists of cancer stations, cancer dispensaries, and hospital cancer departments. At the cancer stations, the public is offered advice and education for the prevention and early detection of malignant disease, while mass screenings are organized, cancer cases registered and patients put in touch with expert facilities. Every doctor is required to refor any patient with an obviously or possibly malignant lesion to one of these stations. The cancer dispen- saries, concentrated in the larger towns, are highly specialized and well-staffed institutions the functions of which include not only the application of the surgical and other treatment required but also the assembly and the analysis of data, the training of medical and allied staff, the education of the public and general collaboration with public health authorities in cancer control. The hospital cancer departments concentrate mainly on the purely medical aspects of cancer treatment at a specialized level.

Thus, although in both the U.S.S.R. and in the United Kingdom it is the general practitioner or his equivalent who makes the first contact with a case of malignant disease, the subsequent procedure is very different; in the former country the patient will be treated by a specialized service while, in the latter, he is most likely to be dealt with in the local general hospital with appropriate facilities. The differences between the two services may eventually become less because of the trend in many countries towards the centralization of hospital services. As knowledge increases, the collaboration of many specialists becomes necessary and the cost of equipment mounts; these factors favour the concentration of cancer patients in a few hospitals at regional, or even at national level.

Efficient cancer services cannot be planned or operated without valid statistics, which must relate to morbidity as well as mortality. Thus, some form of cancer registration is essential. A registration or notification system, particularly if combined with an analysis of treatment and survival statistics, is of the greatest value in providing not only an index of therapeutic efficiency but also important material for epidemiological research. In a small, closely knit country such as Denmark or the Netherlands, a single national registry for all forms of malignant disease is possible. In others it may be necessary to work from several national registers; or there may be separate national registers dealing with parti- 
cular types of tumour such as bone sarcoma. It is from such statistical information as that concerned with the rapid increase of morbidity and mortality from lung cancer in men that ætiological conclusions-and preventive action-may develop.

The prevention of cancer has found its most obvious application in many countries in the attack on causative occupational and industrial factors. Many different carcinogens are encountered in the chemical industry, mining and other occupations; and certain components of polluted air in cities and food additives are known or suspected to be carcinogenic. Ionizing radiation is another potential source of malignancy. Measures to prevent the pollution of air, particularly by benzpyrene, and to limit the hazards of radiation are now established elements of public health service practice in many countries.

Another approach to prevention is through the mass screening of the population to detect precancerous lesions. In most countries of western Europe, such screening is carried out by particular industries or professional groups on a private basis. In eastern European countries and the U.S.S.R., however, more and more extensive examinations of both working and non-working populations are performed each year; this has resulted in the detection of precancerous lesions-particularly of the uterine cervix, breast, skin and stomach-and the early recognition of actual neoplasms. Experience in the U.S.S.R. has demonstrated the importance of planning such surveys carefully. Examinations of persons more than 30-35 years of age are conducted by teams of specialists, and other diseases are looked for simultaneously. Regular radiographic examinations of the chest, and sometimes of the upper gastro-intestinal tract, is also a feature.

In some countries the approach is through examina. tion of all patients attending factory or hospital out-patients clinics for whatever reason. The mass gynæcological examination of women in factories and hospitals is also valuable in connexion with tumours of the cervix and pelvis. In some areas, cytological diagnosis is being practised on a large seale, and this method holds great promise.

One of the greatest problems in obtaining good results from cancer treatment is that of seeing patients at a sufficiently early stage of the disease. Only too often delays constitute a formidable handicap to success. The time-interval between the earliest symptoms and radical treatment may be several months, even for the common cancers of the breast and stomach. In many cases cerebral tumours do not come to operation for a year or 18 months after the first headache. Early diagnosis is achieved partly by educating the public (and the general practitioner) to be aware of, and willing to report, possible symptoms; and partly by providing suitable facilities for diagnosis. A number of European countries has special diagnostic centres, appropriately equipped, attached to hospitals; and in the U.S.S.R. there are the designated cancer dispensaries or polyclinics. A widespread application of the technique of cytological diagnosis may perhaps prove a valuable aid to early diagnosis. This has already shown itself, in some parts of the world, to be of first importance in detecting early cancer of the cervix by means of a simple vaginal smear.

A general development of mass screening and early diagnostic measures depends on sound education of the general public in the issues at stake; yet this is often neglected. Controlled surveys have shown that public attitudes can be changed by campaigns, particularly those directed against specific tumours, the most important effect often being to overcome a feeling of hopelessness about cancer treatment in general. In the U.S.S.R. and in eastern European countries such education is a responsibility of the Ministry of Health; there are institutes of health education, and mass examinations are carried out in factories, all propaganda media being harnessed for the purpose. In other countries this task is left to national societies dealing either with cancer in general or with particular lesions. Cancer of the lung is one of these, and many unofficial bodies are now aiming at the prevention of smoking in school-children and young people. It is interesting to note that, while a leading British authority stresses the risks of imparting too much clinical information to laymen, the view in the U.S.S.R. is that clear and detailed information on every aspect of the problem is the best way of avoiding cancerophobia.

In most countries there is no special legislation governing cancer diagnosis and treatment facilities. In some, where the problem is handled at ministerial level, appropriate regulations exist, for example, in Czechoslovakia and France, where notification is compulsory. Whether special legislation is required is mainly determined by the adequacy of the existing general health legislative framework. In all cases, the objects must include efficient prevention, diagnosis, treatment, after-care and rehabilitation irrespective of economic status; the accumulation of reliable statistics; and the provision for cancer research.

\section{COMPOSITION OF 'PEARLS'}

\section{By Prof. K. VOZOFF, R. M. ELLIS* and Prof. G. D. GARLAND Department of Physics, University of Alberta}

$\mathrm{R}$ ECORDS of micropulsations of the Earth's magnetic field at times exhibit 'pearls' which appear to result from mixing two sinusoidally varying magnetic fields having slightly different frequencies and roughly equivalent amplitudes. Typically these frequencies lie in the range $0.2-2$ c.p.s. and may differ from one another by 0.01-0.1 c.p.s. An identical wave form could be obtained through amplitude modulation of a single oscillator.

In the course of our magnetotelluric investigations a recording containing about $\mathbf{l} \mathrm{hr}$. of impure pearls

* Petroleum Research Fund Fellow. was analysed. The recording of the two horizontal electric components and the three magnetic components was made between $1430 Z$ and $1520 Z$, on September 7, 1961, at a location 20 miles north of Edmonton, Alberta. The average zero crossing period of the pulsations was about $1 \cdot 4-1.5$ sec. Their modulating envelope appeared to bea sinusoid of about 26 sec. period which was itself being modulated. There was a pronounced tendency for the entire pattern to repeat itself at periods of about 145 sec., or after 10 'pearls'. The entire pattern was superposed on much slower variations. Polarization in the horizon- 